\title{
Large-scale velocity flow in the Horologium-Reticulum supercluster
}

\author{
Matthew C. Fleenor ${ }^{1}$, James A. Rose ${ }^{1}$, Wayne A. Christiansen ${ }^{1}$, \\ Richard W. Hunstead ${ }^{2}$, Melanie Johnston-Hollitt ${ }^{3}$ \\ and Michael J. Drinkwater ${ }^{4}$ \\ ${ }^{1}$ Department of Physics \& Astronomy, University of North Carolina, Chapel Hill, North \\ Carolina 27599-3255 USA email: fleenor2@physics.unc.edu \\ ${ }^{2}$ School of Physics, University of Sydney, NSW 2006, Australia \\ ${ }^{3}$ Leiden Observatory, NL-2300 RA Leiden, The Netherlands \\ ${ }^{4}$ Department of Physics, University of Queensland, QLD 4072, Australia
}

\begin{abstract}
We present results from 547 optical galaxy redshifts obtained in the region of Horologium-Reticulum (HR) using the $6 \mathrm{dF}$ (six-degree field) multi-fiber spectrograph on the United Kingdom Schmidt Telescope. Concentrating upon the inter-cluster regions of the HR Supercluster (HRS), we derive three primary results from our $6 \mathrm{dF}$ observations. First, the HRS covers a redshift range from at least 16000 to $22500 \mathrm{~km} \mathrm{~s}^{-1}$. Second, these HRS galaxies exhibit an overall gradient with increasing redshift along a spatial axis of SE-NW with high statistical probability. Third, along the best-fit line from the spatial-redshift linear regression, HRS galaxies comprising the redshift trend are distinctly split into a high- and low-redshift component. Finally, comparisons of the spatial-redshift distribution are made between the HRS and the Shapley supercluster.
\end{abstract}

\section{The Horologium-Reticulum Supercluster (HRS)}

Superclusters of galaxies provide a unique environment for investigating the growth of and inter-relationship between structures on all scales. These massive associations present the opportunity to assess whether structure is indeed assembling in a hierarchical fashion. Covering an area of more than $12^{\circ} \times 12^{\circ}$ on the sky at approximately $\alpha=03^{h} 19^{m}$ and $\delta=-50^{\circ} 02^{\prime}$, the HRS is the second largest mass concentration within $300 \mathrm{Mpc}$, only exceeded in mass by the Shapley Supercluster (SSC).

To obtain a comprehensive picture of the variety of structures present in the HRS complex, we have utilized a combination of the AAT/2dF for investigations of Abell clusters A3125/A3128 and the UKST/6dF for observations of the inter-cluster regions. To probe the finer scale structures within the A3125/A3128 intra-cluster medium, we have also examined radio and X-ray imaging (Rose et al. 2002). Currently, our collaboration is involved in the analysis of extended radio emission in A3125 as it relates to the supercluster environment (Johnston-Hollitt et al. 2004). Here, we summarize several key results concerning the distribution of megaparsec-scale structure extracted from our $6 \mathrm{dF}$ inter-cluster observations.

\subsection{Kinematic extent of the HRS}

The redshift histograms in Figure 1 (left) show the connection between the $6 \mathrm{dF}$ galaxies and clusters of known redshift within the observing region. The majority of $6 \mathrm{dF}$ galaxies (53\%) occupy the redshift range between 16000 and $22500 \mathrm{~km} \mathrm{~s}^{-1}$. The histogram of cluster mean redshifts further supports the above kinematic boundaries for the HRS as most of the clusters fit between the above redshift range (Fig. 1c, left). 

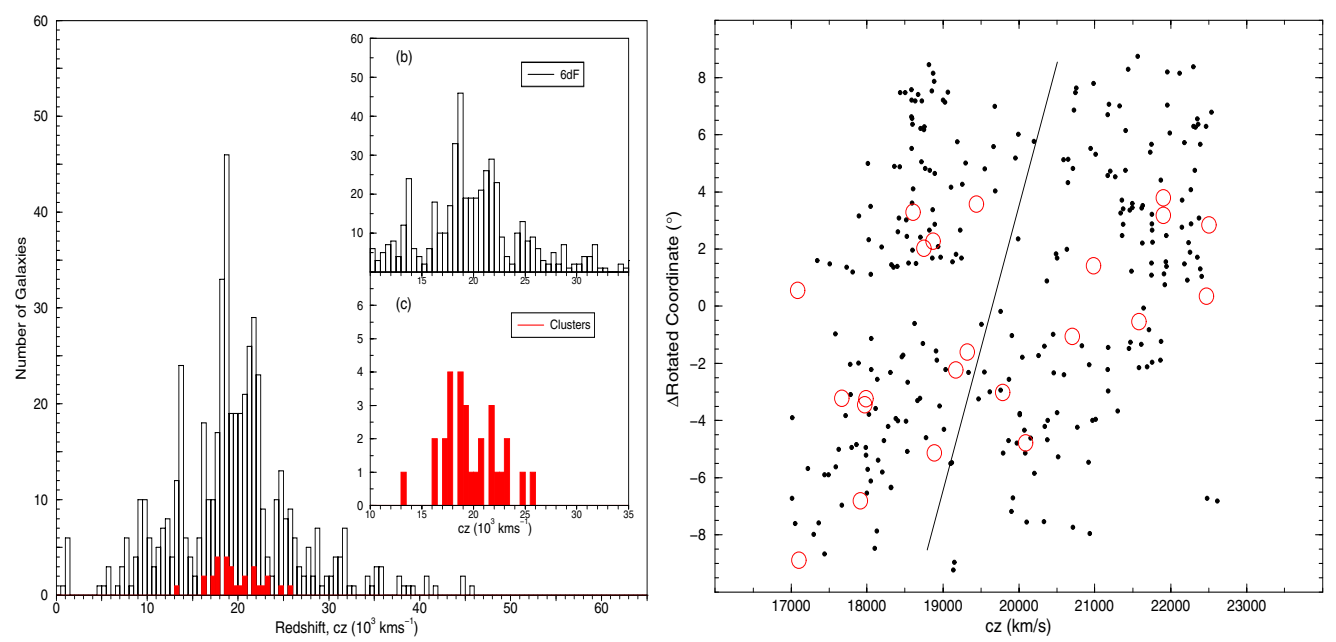

Figure 1. left: Histograms of the $6 \mathrm{dF}$ inter-cluster galaxies (solid lines) and of the clusters with known redshifts (filled histogram) are overlaid. In panels (b) and (c) we show an expanded view of the redshift histograms for the inter-cluster galaxies and the clusters respectively. The redshift bin size is $500 \mathrm{~km} \mathrm{~s}^{-1}$. right: Spatial-redshift plot for $6 \mathrm{dF}$ galaxies between 17000 and $22500 \mathrm{~km} \mathrm{~s}^{-1}$ (center located at $\alpha=3^{h} 16^{m}, \delta=-52^{\circ}, \mathrm{PA}=-70^{\circ}$ ). Open circles represent Abell clusters at an equivalent PA. Solid line is the best-fit line from the linear regression analysis.

\subsection{Large-scale redshift trend}

Qualitative examination of spatial plots of $6 \mathrm{dF}$ galaxies suggests an overall trend between spatial position and redshift, that is, there appears to be a trend of systematically increasing redshift along a principal axis extending from the SE to the NW end of the HRS. To quantify the notion of a systematic redshift trend for $6 \mathrm{dF}$ galaxies between 16000 and $22500 \mathrm{~km} \mathrm{~s}^{-1}$, we have conducted a linear correlation analysis between projected galaxy position and redshift. At consecutive $10^{\circ}$ offsets in position angle (PA), we have projected the galaxy positions onto the principal axis through that PA. We find a significant correlation (with the null hypothesis of no correlation rejected at a high level) for a PA at $-20^{\circ}$ (as measured east from north). However, when the spatially-compact galaxies between 16000 and $17000 \mathrm{~km} \mathrm{~s}^{-1}$ are excluded from the trend, the probability of correlation increases by three orders of magnitude $(>99.9 \%)$. Furthermore, the clusters and galaxies from 17000 to $22500 \mathrm{~km} \mathrm{~s}^{-1}$ share the same best-fit line along the PA of $-70^{\circ}$ after the low-redshift galaxies are excluded. The results of this analysis are summarized in Figure 1 (right) where the projected spatial coordinate is plotted against the redshift. In short, there is a large-scale kinematic trend in the HRS of increasing redshift for galaxies and clusters between 17000 and $22500 \mathrm{~km} \mathrm{~s}^{-1}$ along a SE-NW axis.

\subsection{Bi-modality of the HRS}

After fitting and removing the position-redshift tilt described above, a bi-modal redshift distribution of nearly equal populations emerges for the inter-cluster galaxies (Fig. 2, left). Both redshift components follow the overall trend and are separated by $\sim 2500 \mathrm{~km} \mathrm{~s}^{-1}$. The spatial-redshift trend of the inter-cluster galaxies (Fig. 1, right) displays a similar arrangement to that found in N-body simulations, whereby tidal encounters between equal-mass clusters create spatial-kinematic tilts only after the two clusters have passed through each other. Lastly, we note that the distribution of galaxy cluster mean 

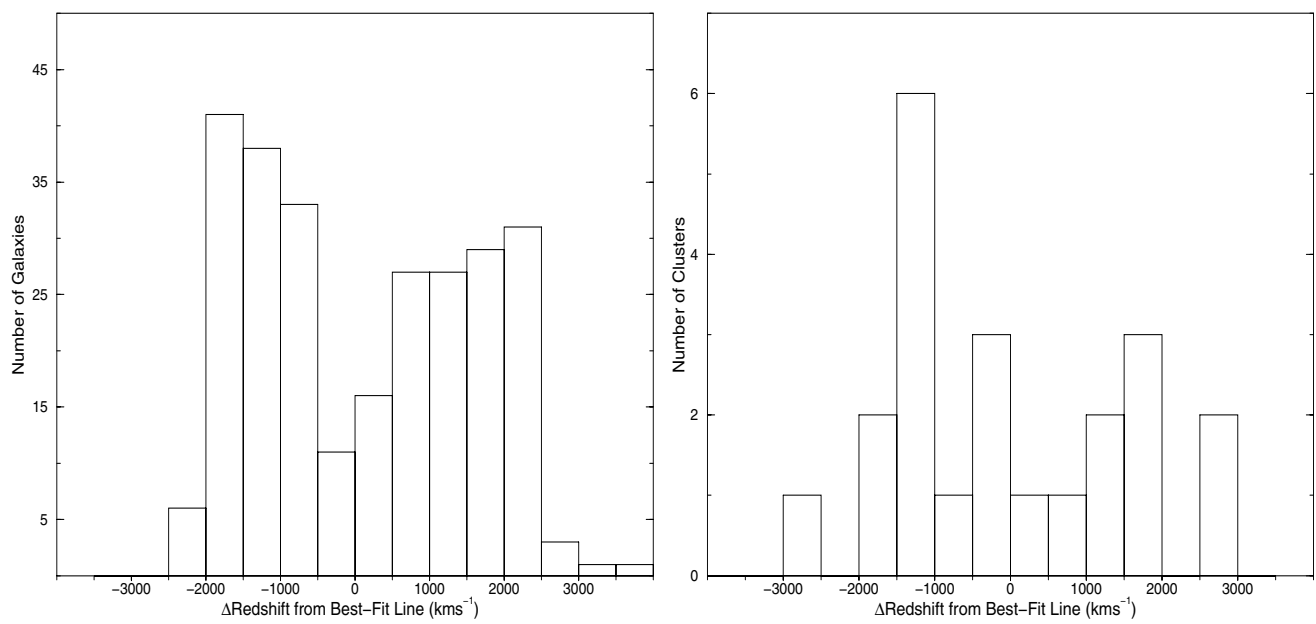

Figure 2. Redshift histograms for galaxies and clusters along the line of best-fit $\left(\mathrm{PA}=-70^{\circ}\right.$, Fig. 1, right). left: $6 \mathrm{dF}$ galaxies. right: Clusters that are given as open circles in Figure 1, right.

redshifts in the HRS does not appear to reflect the bi-modality of the inter-cluster galaxies (Fig. 2, right).

\section{Comparisons with the Shapley concentration}

Since the SSC is both extremely well-studied and the most comparable supercluster in the local universe, we use it as a benchmark for assessing the properties of the HRS. The HRS redshift range from 16000 to $22500 \mathrm{~km} \mathrm{~s}^{-1}$ is comparable to the "Central Region" of the SSC at $\sim 5500 \mathrm{~km} \mathrm{~s}^{-1}$ (Quintana et al. 2000). Bi-modality in redshift is also a feature emerging from inter-cluster studies in the SSC (c.f. Fig. 6 of Drinkwater et al. 1999). However, the redshift peaks in the HRS are nearly equal in amplitude (Fig. 2, left), while the higher redshift peak in the SSC has roughly three times the amplitude of the lower redshift component. Although Bardelli et al. (2000) have fit a plane in $(\alpha, \delta, \mathrm{cz})$ space to their inter-cluster observations in the SSC, this result assumes the redshift is merely a distance and is not readily comparable to the spatial-redshift trend detected in the HRS. Comparisons like these mentioned serve to extricate the individual merging histories of specific superclusters from the dark-matter signatures present in all large-scale structures.

\section{Acknowledgements}

M.C.F. thanks the UNC Graduate School and the AAS for their generosity in providing travel funds. A portion of this work is supported by NSF grant, AST9900720.

\section{References}

Bardelli, S., Zucca, E., Zamorani, G., Moscardini, L., Scaramella, R. 2000, MNRAS, 312, 3

Drinkwater, M. J., Proust, D., Parker, Q. A., Quintana, H., Slezak, E. 1999, PASA, 16, 2

Johnston-Hollitt, M., Fleenor, M. C., Hunstead, R. W., Rose, J. A., Christiansen, W. A. 2004, Proc. IAU Coll. No. 195, A. Diaferio, ed.

Quintana, H., Carrasco, E. R., Reisenegger, A. 2000, AJ, 120, 511

Rose, J. A., Gaba, A. E., Christiansen, W. A., Davis, D. S., Caldwell, N., Hunstead, R. W., Johnston-Hollitt, M. 2002, AJ, 123, 3 